\title{
DATAÇÃO RADIOCARBÔNICA DE SÍTIOS ARQUEOLÓGICOS DO TIPO SAMBAQUI PELA TÉCNICA DE ABSORÇÃO DE CO: UMA ALTERNATIVA À SÍNTESE BENZÊNICA
}

Maria Lúcia T. G. Mendonça

Departamento de Química, Pontifícia Universidade Católica do Rio de Janeiro, Rua Marquês de São Vicente 225, 22453-900 Rio de Janeiro - RJ

José Marcus Godoy*

Instituto de Radioproteção e Dosimetria, Comissão Nacional de Energia Nuclear, CP 37750, 22642-970 Rio de Janeiro - RJ

Recebido em 29/4/03; aceito em 16/7/03

\begin{abstract}
RADIOCARBON DATING OF SAMBAQUI ARCHAEOLOGICAL SITES USING A $\mathrm{CO}_{2}$ ABSORPTION TECHNIQUE: AN ALTERNATIVE TO THE BENZENE SYNTHESIS: The sambaquis are archaeological sites with remains of pre-historical Brazilian civilizations. They look like small hills containing different kinds of shells, animal and fish bones, small artifacts and even human skeletons. Since the sambaqui sites in the Rio de Janeiro state are younger than 6000 years, the applicability of $\mathrm{CO}_{2}$ absorption on Carbo-Sorb ${ }^{\circledR}$ and ${ }^{14} \mathrm{C}$ determination by counting on a low background liquid scintillation counter was tested. The International Atomic Energy Agency standard reference material IAEA-C2 was used in order to standardize the method. Nine sambaqui samples from five different archaeological sites found in the Rio de Janeiro state were analyzed and ${ }^{14} \mathrm{C}$ ages between 2100 and 3600 years BP were observed. The same samples were sent to the ${ }^{14} \mathrm{C}$ Laboratory of the Centro de Energia Nuclear na Agricultura (CENA/USP) where similar results were obtained.
\end{abstract}

Keywords: sambaquis; ${ }^{14} \mathrm{C}$ dating; liquid scintillation counting.

\section{INTRODUÇÃO}

De modo a aumentar a aplicabilidade da técnica de datação baseada no decaimento do ${ }^{14} \mathrm{C}$, através da medição da cintilação em meio líquido, foram realizados esforços no sentido de aumentar a massa de carbono incorporada na solução cintiladora, bem como no de maximizar a relação sinal-ruído empregando-se frascos com baixo teor de potássio, otimizando-se a região de contagem e utilizando-se contadores de cintilação em meio líquido de baixo nível de radiação de fundo.

A solução encontrada para o aumento da massa de carbono, incorporada na solução cintiladora, foi a introdução da síntese benzênica, cujo procedimento detalhado pode ser encontrado no trabalho de Pessenda e Camargo ${ }^{1}$. O emprego da síntese benzênica associada à utilização de contadores de cintilação em meio líquido de baixo nível de radiação de fundo permite trabalhar-se com amostras de até 40.000 anos de idade 2 .

Sambaqui significa, na língua tupi, um morro de conchas. Os sambaquis são sítios arqueológicos com achados de civilizações préhistóricas brasileiras. Assemelham-se a pequenos morros de conchas de ostras e moluscos, contendo ossos de peixes e de pequenos animais, pequenos artefatos e, até, esqueletos humanos. Dada a dieta alimentar destes povos, os sambaquis são encontrados, principalmente, na região costeira. De acordo com Amador $^{3}$, os sambaquis do Estado do Rio de Janeiro possuem idades inferiores a 6.000 anos.

$\mathrm{O}$ método de absorção de $\mathrm{CO}_{2}$ foi desenvolvido como uma alternativa para síntese benzênica, que envolve uma linha de preparação de amostra mais complexa. O método de absorção de $\mathrm{CO}_{2}$ é muito utilizado para amostras que apresentam uma atividade de ${ }^{14} \mathrm{C}$ elevada, em investigações biológicas e hidrogeológicas, mas sua aplicabilidade tem sido testada em amostras que apresentam atividade baixa, devido à sua rapidez e simplicidade na preparação da amostra ${ }^{4-6}$.

*e-mail: jmgodoy@ird.gov.br
O método consiste, primeiramente, num pré-tratamento da amostra, que é semelhante ao da síntese benzênica, na conversão do carbono presente na amostra em $\mathrm{CO}_{2}$, seguida da sua absorção no CarboSorb $^{\circledR}$. Este, contendo $\mathrm{CO}_{2}$, é misturado com a solução cintiladora, seguindo, então, a determinação da atividade de ${ }^{14} \mathrm{C}$ em um detector de cintilação em meio líquido de baixa radiação de fundo ${ }^{4,5}$.

Limites de detecção na faixa de 21.000-29.000 anos BP, ou até mesmo inferior, para a datação baseada na absorção do $\mathrm{CO}_{2}$ têm sido publicados na literatura ${ }^{4,5}$. Dada a idade esperada para os sambaquis existentes no estado do Rio de Janeiro, resolveu-se estudar a aplicabilidade desta técnica na datação deste tipo de amostra, em particular, em face da aquisição de um contador de cintilação em meio líquido de baixa radiação de fundo Packard Tricarb 3170 TR/SL.

\section{PARTE EXPERIMENTAL}

\section{Locais de coleta}

Para demonstrar a aplicabilidade do método proposto, foi realizada a coleta de amostras de conchas de sítios do tipo sambaqui de Sernambetiba, Imenezes, Arapuan, Rio das Pedrinhas e Amorins, que se localizam no Município de Guapimirim, Estado do Rio de Janeiro. Cabe ressaltar que o sambaqui de Amorins foi completamente destruído sendo, portanto, este trabalho a última e única oportunidade de datá-lo. As amostras foram coletadas em conjunto com uma equipe de pesquisadores do Museu Nacional/UFRJ, sob a supervisão da Profa. R. A. R. Peres da Paz.

\section{Pré-tratamento}

De modo a verificar a ocorrência ou não de recristalização, foram separadas as conchas de moluscos das conchas de ostras. O carbonato de cálcio das conchas de moluscos cristaliza-se na forma de aragonita, passando a calcita quando de uma recristalização ${ }^{5}$. Por 
outro lado, as conchas de ostras cristalizam-se na forma de calcita, não sendo possível, desta forma, avaliar-se a ocorrência ou não de recristalização. As conchas foram lavadas com água destilada e deionizada. Com o auxílio de espátula e escova, foram removidos a terra e o material orgânico que se encontravam incrustados nas amostras. As amostras foram colocadas no banho com ultra-som por 1 a $2 \mathrm{~h}$, para remover todas as impurezas que, por ventura, ainda se encontrassem nas reentrâncias das conchas. Novamente, foram lavadas com água destilada e deionizada com auxílio de espátula e escova.

Para remover o carbonato de cálcio secundário, foi utilizado ácido clorídrico a $4 \%$, depois o material foi novamente lavado com água destilada e deionizada. As amostras foram colocadas na estufa por $24 \mathrm{~h} \mathrm{a} 60^{\circ} \mathrm{C}$ para serem secas. Finalmente, foram reduzidas a pó através do uso de gral e pistilo em ágata.

\section{Geração e absorção de $\mathrm{CO}_{2}$}

A quantidade de amostra ideal para ser utilizada no método proposto foi calculada com base na porcentagem de carbonato existente nas conchas, que foi determinada por calcinação a $1000{ }^{\circ} \mathrm{C}$, até massa constante.

Como as amostras consistiam-se $100 \%$ de carbonato de cálcio e, como cada $11 \mathrm{~mL}$ de Carbo-Sorb ${ }^{\circledR}$ absorve até $3,0 \mathrm{~g}$ de $\mathrm{CO}_{2}$, equivalentes a $6,8 \mathrm{~g}$ de carbonato de cálcio, resolveu-se trabalhar com $10 \mathrm{~g}$ de amostra, a fim de garantir a saturação.

Pesaram-se $10 \mathrm{~g}$ de amostra, que foram transferidas para um balão de fundo redondo de três bocas ( $\mathrm{n}^{\mathrm{o}} \mathrm{IV}$ da Figura 1), contendo um bastão magnético. $\mathrm{O} \mathrm{CO}_{2}$ liberado, através da adição de ácido fosfórico $85 \%$ ( ${ }^{\circ} \mathrm{V}$ da Figura 1), foi absorvido em $11 \mathrm{~mL}$ de CarboSorb $^{\circledR}$, que se encontrava num borbulhador ( $n^{\circ} \mathrm{X}$ da Figura 1$)$ imerso num banho de gelo. Uma torneira ( ${ }^{\circ}$ VII da Figura 1) foi utilizada para controlar a entrada de $\mathrm{CO}_{2}$ no meio. Antes da colocação de Carbo-Sorb ${ }^{\circledR}$ no borbulhador, fez-se passar nitrogênio, para retirar todo $\mathrm{CO}_{2}$ atmosférico do mesmo, fechou-se a torneira e colocou-se o Carbo-Sorb ${ }^{\circledR}$ pela outra abertura do recipiente.

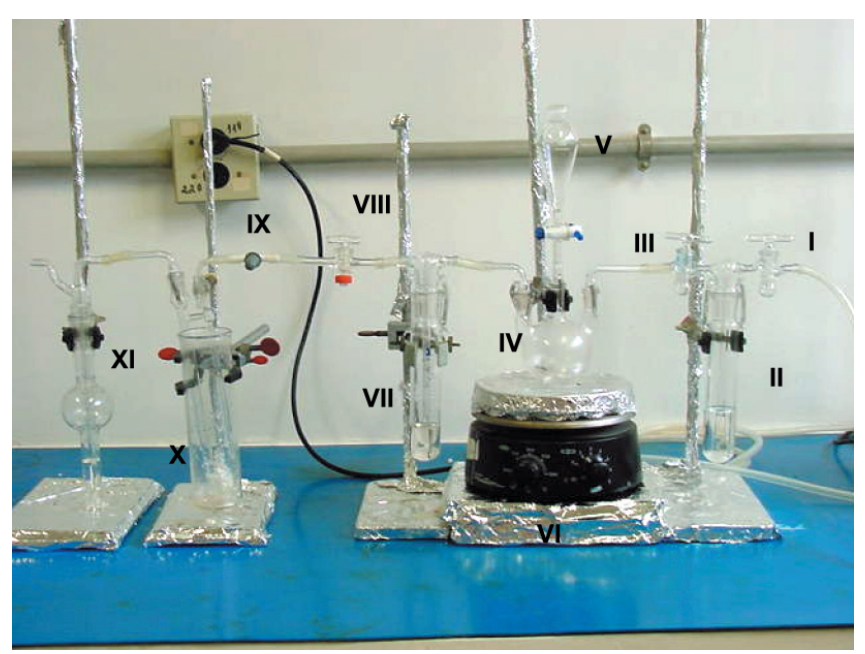

Figura 1. Aparelhagem em vidro empregada na geração e absorção de $\mathrm{CO}_{2}$ proveniente de amostras ricas em carbonatos (I, III e VIII - Torneiras, II e $X I$ - Frasco lavador contendo $\mathrm{NaOH} 1 \mathrm{M}, \mathrm{IV}$ - Balão de fundo redondo de 3 bocas, $V$ - Funil de separação contendo $\mathrm{H}_{3} \mathrm{PO}_{4}$ conc., VI - Agitador magnético, VII - Frasco lavador contendo $\mathrm{H}_{2} \mathrm{SO}_{4}$ conc., IX - Septo, XBorbulhador para absorção de $\mathrm{CO}_{2}$ )

A contaminação com $\mathrm{CO}_{2}$ atmosférico foi minimizada através da existência de um primeiro frasco ( $\mathrm{n}^{\circ}$ II da Figura 1), contendo hidróxido de sódio $1 \mathrm{M}$, evitando que qualquer $\mathrm{CO}_{2}$ que, porventura, estivesse presente no cilindro de nitrogênio, viesse contaminar a aparelhagem. As torneiras ( ${ }^{\circ}$ I e III da Figura 1 ) tinham como objetivo controlar o fluxo de nitrogênio no meio. Antes da adição do ácido fosfórico, toda a aparelhagem era purgada com nitrogênio gasoso por cerca de $5 \mathrm{~min}$.

$\mathrm{O}$ frasco $\mathrm{n}^{\mathrm{o}}$ VII da Figura 1 continha $\mathrm{H}_{2} \mathrm{SO}_{4}$ concentrado como dessecante, já que a reação do ácido fosfórico com a amostra libera água, sendo esta retida neste frasco. A agitação magnética $\left(n^{\circ}\right.$ VI da Figura 1) foi acionada somente após todo o ácido fosfórico ter sido adicionado, de modo que a reação se processasse bem lentamente. A agitação foi realizada até o Carbo-Sorb ${ }^{\circledR}$ tornar-se muito viscoso, não possibilitando mais a absorção do $\mathrm{CO}_{2}$ gerado. A massa de $\mathrm{CO}_{2}$ absorvida no Carbo-Sorb ${ }^{\circledR}$ foi determinada gravimetricamente.

No final do processo, somente, $10 \mathrm{~mL}$ de Carbo-Sorb ${ }^{\circledR}$ saturado foi transferido para um frasco de contagem (vial), ao qual adicionou-se $10 \mathrm{~mL}$ de Permafluor ${ }^{\circledR}$, fechou-se e agitou-se. Por último, o frasco de contagem foi colocado em geladeira por, no mínimo, $24 \mathrm{~h}$ para eliminar o efeito da luminescência, antes de ser colocado no equipamento de medida.

\section{Determinação da atividade de ${ }^{14} \mathrm{C}$}

Foi empregado um contador de cintilação em meio líquido Packard Tricarb 3170 TR/SL nas seguintes condições de operação: controles de estática e luminescência ativados, pré-contagem de 1 min e tempo de contagem de 1440 min. A eficiência de contagem, nas condições de trabalho estabelecidas, situava-se na faixa de $80 \%$. O grau de atenuação "quenching" era verificado amostra/amostra através da fonte externa do equipamento de medida, este parâmetro (tSIE) também pode ser utilizado na verificação da quantidade de $\mathrm{CO}_{2}$ absorvida ${ }^{4}$.

O método foi padronizado empregando-se o material de referência C-2, carbonato de cálcio, da Agência Internacional de Energia Atômica (IAEA), com teor de ${ }^{14} \mathrm{C}$ igual a $41,14 \pm 0,03 \%$ pMC (percentagem do carbono moderno). As aliquotas tomadas foram submetidas a todo o processo de geração e absorção de $\mathrm{CO}_{2}$, descrito anteriormente.

\section{RESULTADOS E DISCUSSÃO}

Como comentado no item referente à determinação da atividade do ${ }^{14} \mathrm{C}$, é possível utilizar-se o grau de atenuação como modo de determinação da massa de $\mathrm{CO}_{2}$ absorvido ${ }^{4}$. Testou-se tal possibilidade, empregando-se quantidades crescentes de carbonato de cálcio como amostra. A relação linear observada entre a massa de $\mathrm{CO}_{2}$ absorvida, determinada por gravimetria, e o parâmetro tSIE, utilizado como monitor do grau de atenuação "quenching", reforça esta possibilidade (Figura 2).

Após a saturação, $10 \mathrm{~mL}$ de Carbo-Sorb ${ }^{\circledR}$ continha cerca de 0,7 $\mathrm{g}$ de carbono, similar ao observado por outros autores ${ }^{4-6}$. Com base nos valores determinados através do emprego do material de referência IAEA C-2, obteve-se uma eficiência global do método de $(8,60 \pm 0,41) \mathrm{cpm} / \mathrm{gC}_{\text {moderno }}$, este valor foi confirmado empregando-se conchas recentes e $\mathrm{CaCO}_{3}$ produzido a partir da absorção $\mathrm{CO}_{2}$ atmosférico.

A contribuição da radiação de fundo foi determinada empregando-se uma amostra de mármore. Foram realizadas 10 determinações e o valor médio observado foi de $(3,93 \pm 0,16) \mathrm{cpm}$. Empregando-se a eficiência global do método e o desvio padrão observado para a radiação de fundo, calculou-se o limite de aplicabilidade do método em 22,4 mil anos ou o equivalente a 4 tempos de meia vida do ${ }^{14} \mathrm{C}$. Considerando que os sambaquis existentes hoje no Estado do Rio de 


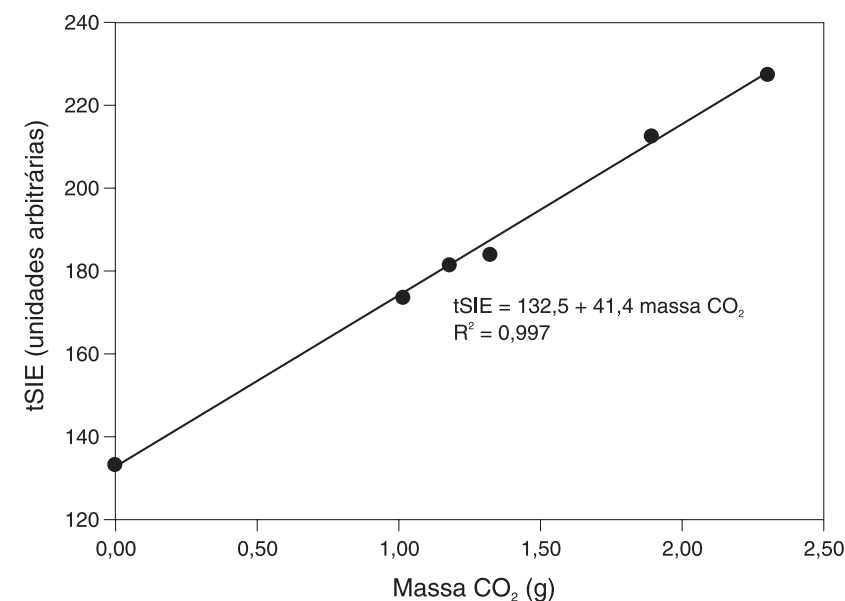

Figura 2. Variação do grau de atenuação “quenching” em função da massa de $\mathrm{CO}_{2}$ absorvida em $10 \mathrm{~mL}$ de Carbo-Sorb ${ }^{\circledast}$

Janeiro possuem idades iguais ou inferiores a 6.000 anos, este limite de idade alcançado é adequado aos objetivos do presente trabalho.

Nove amostras de conchas provenientes dos sítios arqueológicos citados foram datadas empregando-se a metodologia desenvolvida. Alíquotas destas amostras foram enviadas ao Laboratório de ${ }^{14} \mathrm{C}$ do Centro de Energia Nuclear na Agricultura (CENA/USP) para datação. Os resultados obtidos são apresentados na Tabela 1. Ambos resultados referem-se à idade radiocarbônica e foram corrigidos para o efeito de discriminação de massa, com base no $\delta^{13} \mathrm{C}$ determinado pelo CENA/USP. Como pode ser observado, não existe diferença sistemática entre as duas técnicas. A menos das amostras Rio das Pedrinhas e Sernambetiba 3, a diferença entre ambos laboratórios é inferior a $10 \%$.

As idades radiocarbônicas observadas para estes sambaquis, 2000-3500 anos BP, confirmam terem os sambaquis encontrados no Estado do Rio Janeiro uma idade inferior a 6000 anos $^{3}$.

\section{CONCLUSÃO}

Foi possível demonstrar a aplicabilidade de um sistema simples de geração/absorção de $\mathrm{CO}_{2}$ na datação radiocarbônica de amostras ricas em carbonato. $\mathrm{O}$ sistema foi testado em amostras de sambaquis, existentes no Estado do Rio de Janeiro. As idades encontradas varia-
Tabela 1. Tabela comparativa das idades radiocarbônicas de amostras de sambaquis, coletados no município de Magé, Estado do Rio de Janeiro, obtidas pelo método da adsorção de $\mathrm{CO}_{2}$ e pela síntese benzênica (CENA/USP)

\begin{tabular}{lccc}
\hline \multirow{2}{*}{ Amostra } & \multicolumn{2}{c}{ Idades BP } & \\
\cline { 2 - 3 } & $\begin{array}{c}\text { Absorção } \\
\mathrm{CO}_{2}\end{array}$ & $\begin{array}{c}\text { Sintese } \\
\text { Benzênica }\end{array}$ & $\begin{array}{c}\text { Diferença } \\
(\%)\end{array}$ \\
\hline Amorins & $(3350+/-160)$ & $(3540+/-70)$ & $-5,4$ \\
Arapuan 1 & $(2770+/-150)$ & $(2800+/-60)$ & $-1,1$ \\
Arapuan 2 & $(3430+/-160)$ & $(3340+/-70)$ & $-2,7$ \\
Arapuan 3 & $(3180+/-160)$ & $(3460+/-70)$ & $-8,1$ \\
Imenezes & $(2440+/-150)$ & $(2600+/-60)$ & $-6,2$ \\
Rio das Pedrinhas & $(3690+/-160)$ & $(3170+/-70)$ & 16,4 \\
Sernambetiba 1 & $(2110+/-150)$ & $(2160+/-60)$ & $-2,3$ \\
Sernambetiba 2 & $(2190+/-150)$ & $(2290+/-60)$ & $-4,4$ \\
Sernambetiba 3 & $(2820+/-150)$ & $(2510+/-60)$ & 12,4 \\
\hline
\end{tabular}

ram de 2000 a 3500 anos BP, coerentes com o esperado para tal tipo de amostras. As mesmas amostras foram datadas no CENA/USP, tendo sido observadas, em geral, diferenças inferiores a $10 \%$ entre ambos laboratórios.

\section{AGRADECIMENTOS}

Os autores gostariam de agradecer à equipe do Museu Nacional/ UFRJ, em especial, nas pessoas dos Profs. R. A. R. Peres da Paz e B. H. R. Francisco, e à CAPES/MEC pelo apoio financeiro.

\section{REFERÊNCIAS}

1. Pessenda, L. C. R.; Camargo, P. B.; Quim. Nova 1991, 14, 98.

2. Bowman, S.; Interpreting the Past: Radiocarbon Dating, University of Califórnia Press, 1990.

3. Amador, E. S.; Baía da Guanabara e Ecossistemas Periféricos: Homem e Natureza, edição do autor, 1997.

4. Qureshi, R. M.; Aravena, R.; Fritz, P.; Drimmie, R.; Appl. Geochem. 1989, 4, 625 .

5. Nair, A. R.; Sinha, U. K.; Joseph, T. B.; Rao; S. M.; Nucl. Geophys. 1995, 9, 263.

6. Thomson, J. Em Handbook of Radioactivity Analysis; L’Annunziata, M. F., ed.; Academic Press, 1998, cap. 7. 